\title{
Effect of Computer Navigation on Accuracy and Reliability of Limb Alignment Correction following Open-Wedge High Tibial Osteotomy: A Meta-Analysis
}

\author{
Seung-Beom Han, ${ }^{1}$ Hyun Jung Kim, ${ }^{2}$ and Dae-Hee Lee ${ }^{3}$ \\ ${ }^{1}$ Department of Orthopaedic Surgery, Korea University Anam Hospital, Korea University College of Medicine, \\ Seoul, Republic of Korea \\ ${ }^{2}$ Department of Preventive Medicine, Korea University College of Medicine, Seoul, Republic of Korea \\ ${ }^{3}$ Department of Orthopaedic Surgery, Samsung Medical Center, Sungkyunkwan University School of Medicine, \\ Seoul, Republic of Korea
}

Correspondence should be addressed to Dae-Hee Lee; eoak22@empal.com

Received 9 May 2017; Accepted 6 September 2017; Published 9 October 2017

Academic Editor: Amal Khoury

Copyright (C) 2017 Seung-Beom Han et al. This is an open access article distributed under the Creative Commons Attribution License, which permits unrestricted use, distribution, and reproduction in any medium, provided the original work is properly cited.

\begin{abstract}
Background. It is unclear whether computer navigation can improve the accuracy and reliability of targeted lower limb alignment correction following open-wedge high tibial osteotomy (HTO). This meta-analysis was designed to compare the accuracy and reliability of limb alignment correction between computer navigated and conventional open-wedge HTOs. Methods. Studies that compared postoperative coronal alignment, including mechanical axis (MA) and weight bearing line (WBL) ratio, outliers of alignment correction, and change in tibial posterior slope, following open-wedge HTO performed using computer navigated and conventional methods were included. Results. Ten studies were included in the meta-analysis. The MA $\left(0.93^{\circ} ; 95 \%\right.$ confidence interval [CI]: $\left.0.45-1.41^{\circ} ; P<0.001\right)$ and WBL ratio $(1.5 \%$; 95\% CI: $0.03-2.98 \% ; P=0.048)$ were significantly greater for computer navigated HTO than for conventional HTO. Outliers of alignment correction after surgery were significantly lower in patients who underwent computer navigated HTO than in those who underwent conventional HTO (odds ratio: 0.25; 95\% CI: 0.08-0.79; $P=0.02$ ). Changes in posterior tibial slope from before to after surgery, however, were similar for the two approaches. Conclusion. Computer navigated HTO resulted in slightly more valgus postoperative alignment and effectively reduced outliers of alignment correction but had no effect on change in posterior tibial slope when compared with conventional HTO.
\end{abstract}

\section{Introduction}

Open-wedge high tibial osteotomy (HTO) is easier to perform and is more adjustable for alignment correction than closed-wedge HTO; also it has the advantage of avoiding the complications associated with closed-wedge HTO, such as peroneal nerve palsy $[1,2]$. Although accurate alignment correction is a key factor for achieving successful openwedge HTO [3-5], preoperatively planned optimal correction of alignment is difficult to determine during surgery [6], because the lower limb is covered with sterile drapes and wrapped by a tourniquet [7]. Many procedures have been utilized to determine intraoperative alignment correction, such as cable, grids, and a jig-based system under fluoroscopy, but none has proven satisfactory to date.

Computer navigation has been shown to be effective for accurate restoration of neutral alignment in patients undergoing total knee arthroplasty (TKA) [8], suggesting that computer navigation may be utilized to determine the intraoperative adequacy of alignment correction during open-wedge HTO [9]. Unlike TKA, in which alignment is determined by direct osseous contact, the indirect skin contact registration process for bony landmarks in openwedge HTO may lead to different outcomes. In addition, previous studies comparing computer navigated and conventional open-wedge HTOs have shown conflicting results. 
This meta-analysis was therefore designed to compare the accuracy and reliability of limb alignment correction between computer navigated and conventional open-wedge HTOs. It was hypothesized that the two approaches would be similarly accurate and reliable.

\section{Methods}

2.1. Data and Literature Sources. The study design was based on Cochrane Review Methods. Multiple comprehensive databases, including MEDLINE (1 January 1976 to 31 May 2015), EMBASE (1 January 1985 to 31 May 2015), the Cochrane Library (1 January 1987 to 31 May 2015), and KoreaMed (1 June 1958 to 31 May 2015), were searched for studies that compared graft extrusion on postoperative MRI in patients who underwent medial and lateral MATs. There were no restrictions on language or year of publication. Search terms used in the title, abstract, $\mathrm{MeSH}$, and keywords fields included "Osteotomy" [tiab] OR “Tibial” [tiab] OR "High" [tiab] OR "Navigation" [tiab], OR “Open" [tiab], AND "Osteotomy" [MeSH] OR "Computer-assisted" [tiab]. After the initial electronic search, relevant articles and their bibliographies were searched manually. Articles identified were assessed individually for inclusion.

2.2. Study Selection. Study inclusion was decided independently by two reviewers, based on the predefined selection criteria. Titles and abstracts were read; if suitability could not be determined, the full article was evaluated. Studies were included in the meta-analysis if they compared alignment correction in patients who underwent open-wedge HTO with and without computer navigation; they simultaneously reported direct comparisons of computer navigation and conventional HTOs; and their primary outcomes included comparisons of coronal alignment after surgery, including mechanical axis (MA), weight bearing line (WBL) ratio, outliers of coronal alignment, and/or changes in posterior slope from before to after surgery. MA was defined as the angle subtended by a line drawn from the center of the femoral head to the center of the tibial spines and a line drawn from the center of the tibial spines to the center of the talus, and WBL was defined as a line drawn from the center of the femoral head to the center of the superior articular surface of the talus. WBL ratio was calculated as the tibial intersection of the WBL/tibial width, with the medial tibial edge defined as $0 \%$ and the lateral tibial edge as $100 \%$. Outliers of coronal alignment were defined as deviations from the acceptable range or tolerance of the targeted alignment correction. The angle of the posterior tibial slope on knee lateral radiographs was defined as the angle between the proximal plateau and a line drawn perpendicular to the tibial shaft axis. Studies were also included in the meta-analysis if they fully reported the number of knees in each group (computer navigated versus conventional groups), the means and standard deviations of parameters of alignment correction and posterior slope change, and the numbers that deviated from the acceptable range of targeted alignment correction in each group and if they used adequate statistical methods to compare alignment parameters, outliers of alignment correction, and posterior slope change in the two groups.

2.3. Data Extraction. Two reviewers independently recorded data from each study using a predefined data extraction form. Any disagreement unresolved by discussion was reviewed by a third author. Variables recorded included (1) means and standard deviations of postoperative MA or WBL ratio and numbers of outliers of coronal alignment following computer navigated and conventional HTO, (2) complications during or following open-wedge HTO such as lateral tibial cortical fractures and delayed union or nonunion, (3) the sample size of each group, and (4) the type of computer navigation system. If these variables were not mentioned in the articles, the study's authors were contacted by email to request these data.

2.4. Assessment of Methodological Quality. Two reviewers independently assessed the methodological quality of each study using the Newcastle-Ottawa Scale, as recommended by the Cochrane Non-Randomized Studies Methods Working Group. In this study, the Newcastle-Ottawa Scale's star system, which awards stars depending on the level of bias, was adjusted to a scale that included only low (one star), high, and unclear bias. Each study was judged on three criteria: the selection of the study groups, the comparability of the groups, and the ascertainment of either the exposure or outcome of interest for case-control or cohort studies. Any unresolved disagreements between reviewers were resolved by consensus or by consultation with a third investigator.

2.5. Statistical Analysis. The main outcomes of the metaanalysis were the mean differences in MA and WBL ratio, change in posterior slope from before to after surgery, and the proportion of outliers of coronal alignment between groups of knees that underwent computer navigated and conventional HTO. Binary outcomes were reported as odds ratios (ORs) and 95\% confidence intervals (CIs), whereas continuous outcomes comparing medial and lateral MATs were reported as mean differences and 95\% CIs. Heterogeneity was determined by estimating the proportion of between-study inconsistencies due to actual differences between studies rather than differences due to random error or chance, using the $I^{2}$ statistic, with values of $25 \%, 50 \%$, and $75 \%$ considered low, moderate, and high, respectively. All statistical analyses were performed using RevMan version 5.2 and Stata/MP 13.0.

\section{Results}

3.1. Identification of Studies. Figure 1 shows the details of study identification, inclusion, and exclusion. Electronic searches yielded 179 studies in PubMed (MEDLINE), 235 in EMBASE, and 10 in the Cochrane Library. Two additional publications were identified through manual searching. After removing 127 duplications, 299 studies remained; of these, 267 were excluded based on reading of the abstracts and fulltext articles. An additional 20 studies were excluded, since they included subjects who underwent computer navigated 


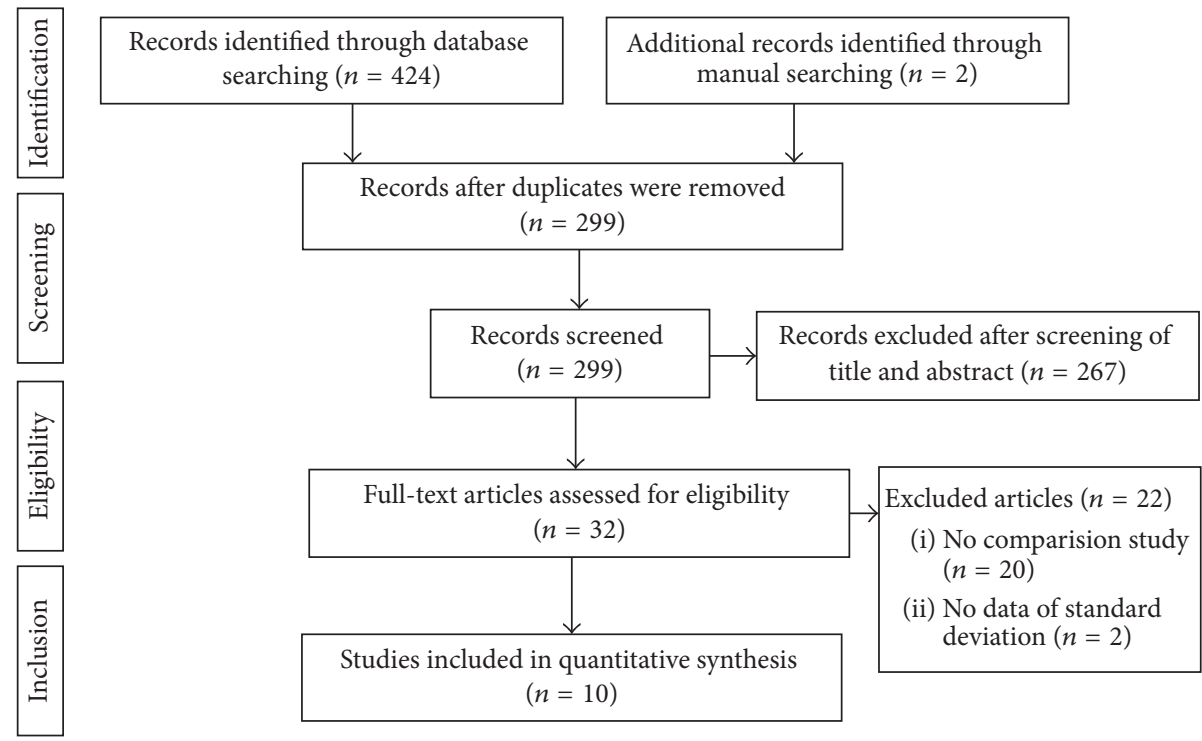

FIgURE 1: PRISMA (Preferred Reporting Items for Systematic Reviews and Meta-analyses) flow diagram of the identification and selection of the studies included in this meta-analysis.

or conventional HTO, not both, and two were excluded because they did not report standard deviations of alignment. After applying these criteria, 10 studies were finally included in this meta-analysis.

3.2. Study Characteristics and Quality Assessment. The 10 included studies involved 275 knees that underwent computer navigated open-wedge HTO and 251 knees that underwent conventional open-wedge HTO. The 10 studies retrospectively compared combinations of four parameters: absolute coronal alignment, including MA and/or WBL ratio, proportion of outliers of alignment correction after openwedge $\mathrm{HTO}$, and change in tibial posterior slope from before to after surgery. Three studies measured three parameters, five compared two parameters, and two compared one parameter each. Four of the 10 included studies reported complications, which included lateral cortex fracture, delayed union, and a broken screw on the plate (Table 1). All ten studies included in this meta-analysis had a low risk of selection bias and compared demographic data between knees that underwent computer navigated and conventional open-wedge HTO; none, however, assessed possible confounding factors. Sufficient follow-up duration was defined as the time from surgery to taking radiographs, with shorter time intervals associated with a higher risk of bias, because early follow-up radiographs could not measure actual alignment correction due to remaining flexion contracture. If postoperative radiographs were taken within three months after surgery, that study was considered as having a high risk of bias. Table 2 summarizes the risk of bias for the 10 studies included in this metaanalysis.

3.3. Coronal Alignment. Of the 10 studies, 9 compared postoperative coronal alignment correction between computer navigated and conventional HTO, as determined by measuring the MA or WBL ratio. These nine studies included 261 subjects who underwent computer navigated HTO and 238 who underwent conventional HTO. Pooled data showed that MA was $0.93^{\circ}$ greater in computer navigated than in conventional HTO (95\% CI: $0.45^{\circ}-1.41^{\circ} ; P<0.001 ; I^{2}=6 \%$ ) and that WBL ratio from the medial edge of the tibial plateau was $1.5 \%$ greater in computer navigated than in conventional HTO (95\% CI: $0.03 \%-2.98 \% ; P=0.048 ; I^{2}=86 \%$, Figure 2). These findings indicated that computer navigated HTO resulted in slightly more valgus coronal alignment than conventional HTO.

3.4. Outliers of Alignment. Of the 10 studies, seven reported the proportion of knees deviated from correction target (MA, $2^{\circ}$ to $8^{\circ}$, or $\mathrm{WBL}$ ratio, $50 \%$ to $70 \%$ ). The pooled results showed that the proportion of postoperative outliers of alignment correction was significantly lower in patients who underwent computer navigated than conventional HTO (32/200 [16.0\%] versus 56/178 [31.5\%]; OR: 0.25; 95\% CI: $0.08-0.79 ; P=0.02 ; I^{2}=69 \%$, Figure 3 ).

3.5. Change of Posterior Tibial Slope. Four studies compared changes from baseline in posterior tibial slope following computer navigated and conventional HTO. These studies included 136 subjects who underwent computer navigated HTO and 131 who underwent conventional HTO. The pooled results showed that the change in posterior tibial slope was $1.13^{\circ}$ smaller with computer navigated than with conventional HTO, but this difference was not statistically significant $(95 \%$ CI: $-3.44^{\circ}-1.18^{\circ} ; P=0.34 ; I^{2}=56 \%$, Figure 4 ). 


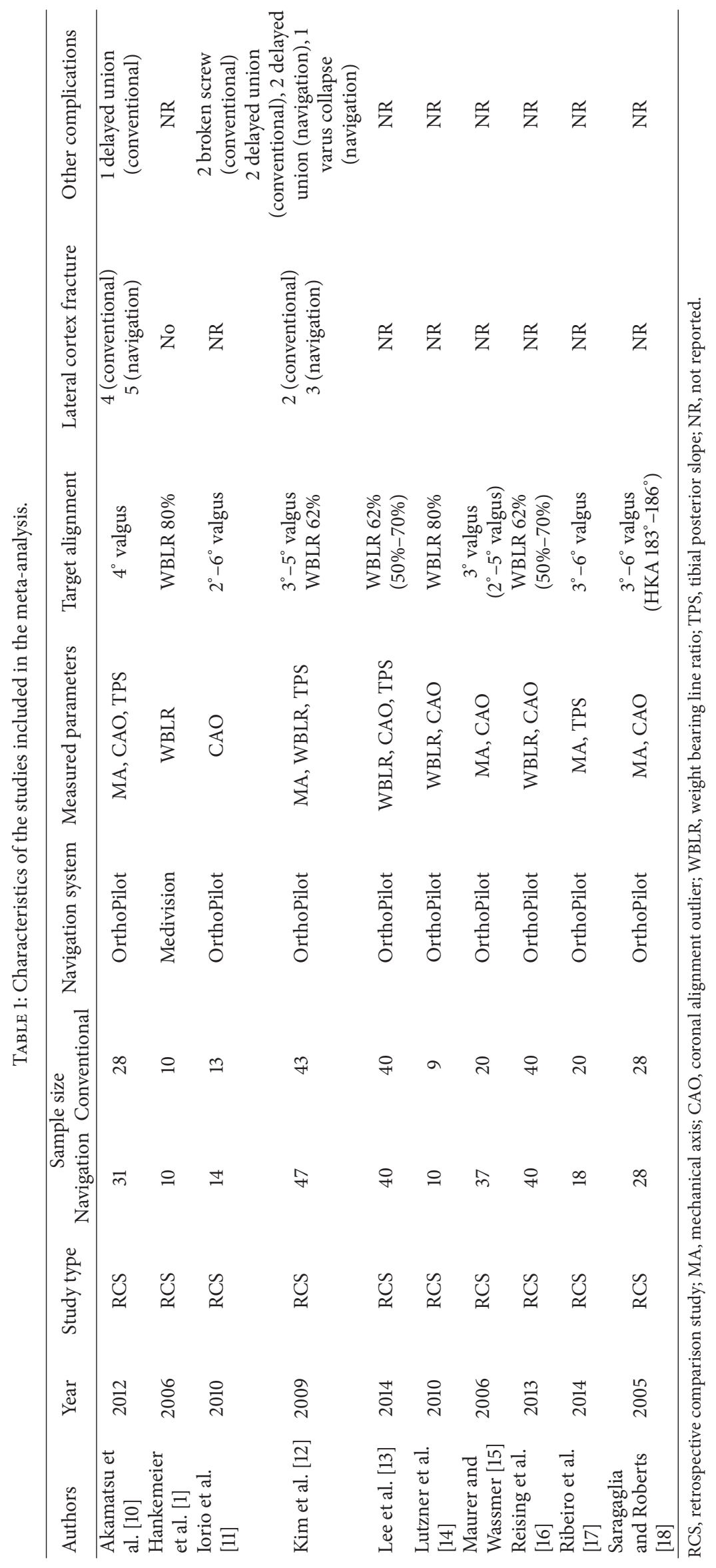




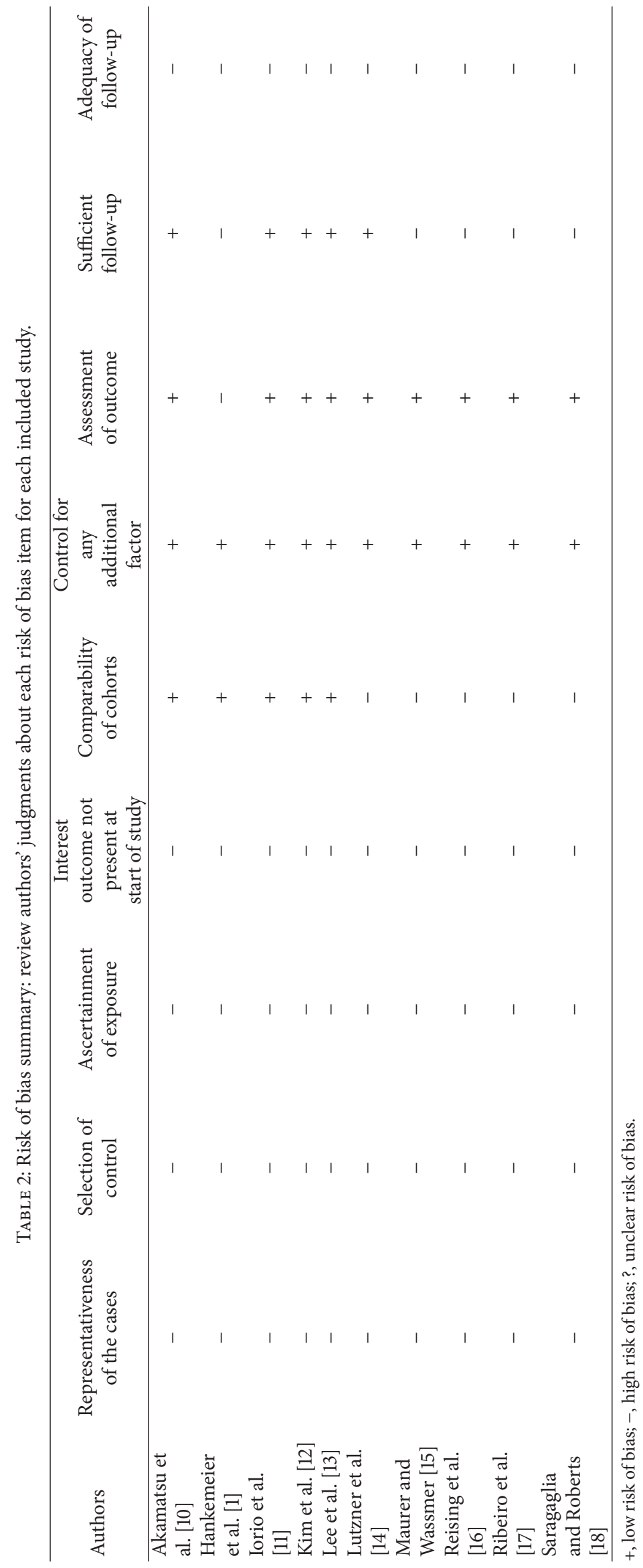




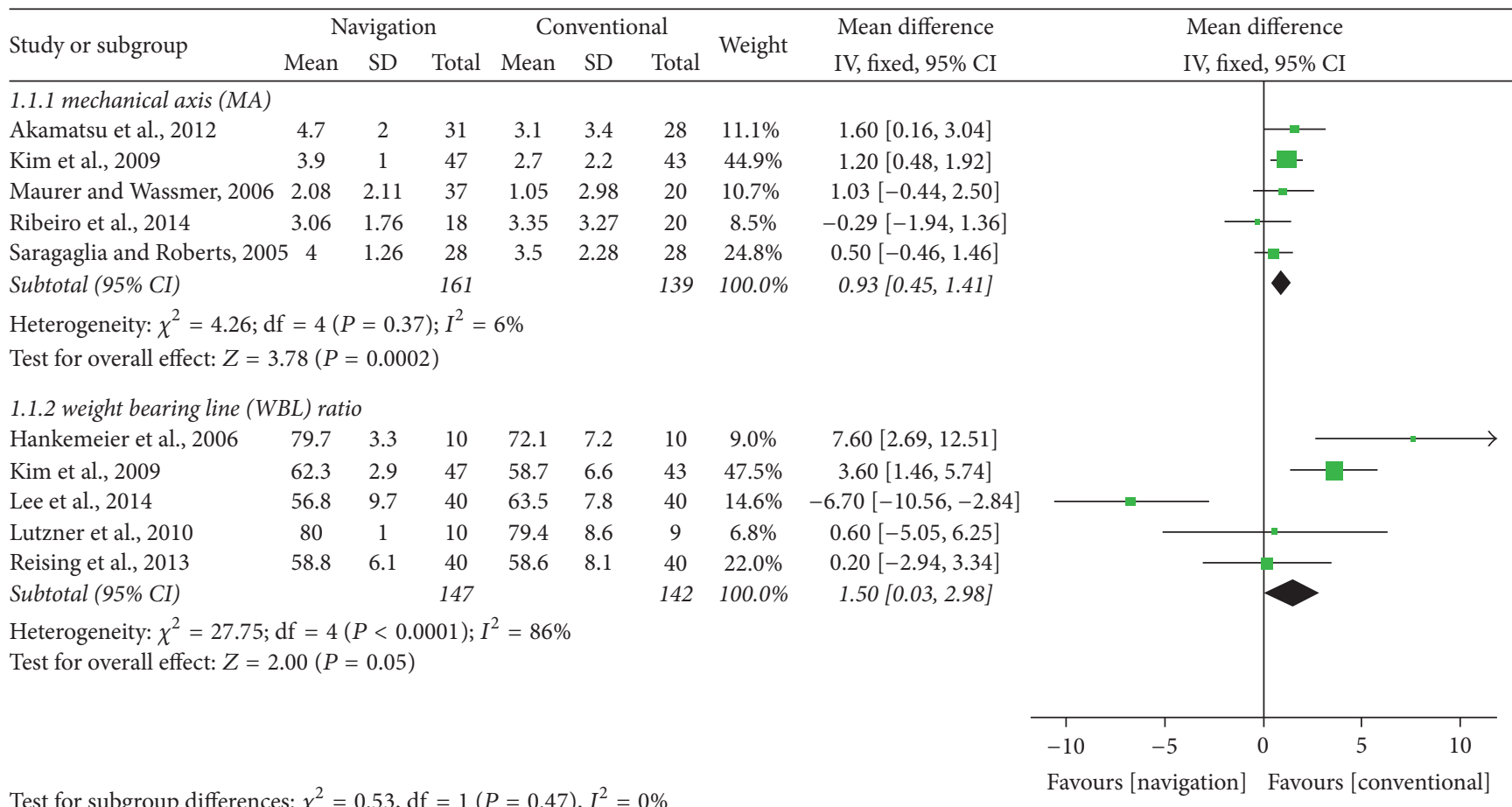

Test for subgroup differences: $\chi^{2}=0.53, \mathrm{df}=1(P=0.47), I^{2}=0 \%$

Favours [navigation] Favours [conventional]

FIGURE 2: Forest plot showing the mean differences in coronal alignments, including mechanical axis and weight bearing line ratio, between computer navigated and conventional high tibial osteotomies.

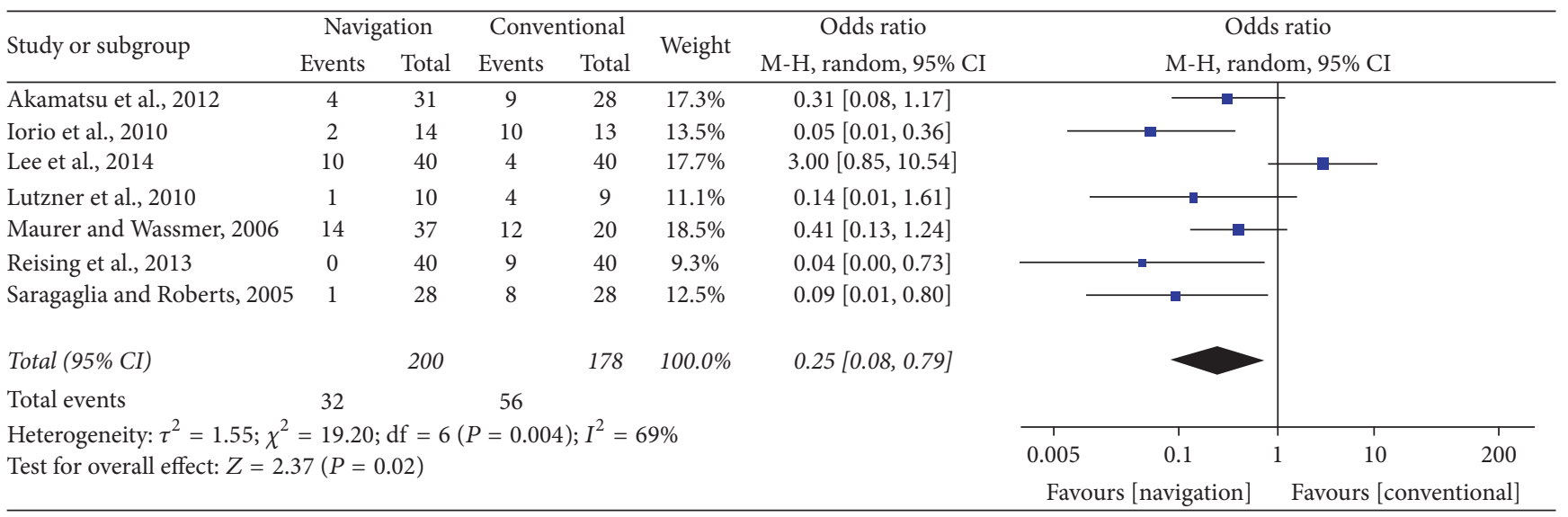

FIGURE 3: Forest plot showing the proportion of outliers of alignment $\left(> \pm 3^{\circ}\right)$ between computer navigated and conventional high tibial osteotomies.

\begin{tabular}{|c|c|c|c|c|c|c|c|c|c|c|c|}
\hline \multirow{3}{*}{$\begin{array}{l}\text { Study or subgroup } \\
\text { Akamatsu et al., } 2012\end{array}$} & \multicolumn{3}{|c|}{ Navigation } & \multicolumn{3}{|c|}{ Conventional } & \multirow{2}{*}{ Weight } & \multirow{2}{*}{$\begin{array}{c}\text { Mean difference } \\
\text { IV, random, 95\% CI }\end{array}$} & \multirow{2}{*}{\multicolumn{3}{|c|}{$\begin{array}{l}\text { Mean difference } \\
\text { IV, random, 95\% CI }\end{array}$}} \\
\hline & Mean & $\mathrm{SD}$ & Total & Mean & $\mathrm{SD}$ & Total & & & & & \\
\hline & 0.6 & 2.1 & 31 & 3.5 & 3.6 & 28 & $39.0 \%$ & $-2.90[-4.42,-1.38]$ & - & & \\
\hline Kim et al., 2009 & 0.5 & 7.6 & 47 & 0.6 & 8.2 & 43 & $24.2 \%$ & $-0.10[-3.38,3.18]$ & & & \\
\hline Lee et al., 2014 & 1 & 8.6 & 40 & -0.5 & 6.6 & 40 & $23.6 \%$ & $1.50[-1.86,4.86]$ & & 要 & \\
\hline Ribeiro et al., 2014 & 1.94 & 7.3 & 18 & 4.4 & 9.5 & 20 & $13.3 \%$ & $-2.46[-7.82,2.90]$ & $\leftarrow$ & & \\
\hline Total (95\% CI) & & & 136 & & & 131 & $100.0 \%$ & $-1.13[-3.44,1.18]$ & & & \\
\hline \multicolumn{9}{|c|}{$\begin{array}{l}\text { Heterogeneity: } \tau^{2}=2.96 ; \chi^{2}=6.79 ; \mathrm{df}=3(P=0.08) ; I^{2}=56 \% \\
\text { Test for overall effect: } Z=0.96(P=0.34)\end{array}$} & -4 & 2 & 4 \\
\hline
\end{tabular}

FIGURE 4: Forest plot showing the mean differences in posterior tibial slope between computer navigated and conventional high tibial osteotomies. 


\section{Discussion}

The most important findings of this meta-analysis were that computer navigated HTO tended to result in more valgus alignment and reduced the percentage of outliers of alignment correction after surgery than did conventional HTO.

Although computer navigated HTO resulted in slightly more valgus alignment correction, it is unclear whether this more valgus tendency was associated with better clinical outcomes. Nine of the 10 studies included in this meta-analysis used the same computer navigation system (OrthoPilot), with a targeted correction of alignment of $30-40 \%$ of the lateral tibial plateau from the center of the knee [19]. In contrast, conventional preoperative planning targeting the amount of correction aimed for the mechanical axis to pass $62.5 \%$ laterally from the medial tibial plateau margin [20]. If the medial and lateral tibia plateaus are symmetrical, the target point of the computer navigation system would be $65-70 \%$ from the medial tibial plateau margin or $2.5-7.5 \%$ more lateral than the target point of preoperative planning [15]. This difference in target points may account in part for the slightly more valgus postoperative alignment in computer navigated than conventional open-wedge HTO.

Another possible reason that computer navigated HTO resulted in a slightly more valgus alignment may be due to differences in weight bearing conditions [21]. Computer navigated HTO measures alignment with the patient in the supine position, without weight bearing. Following osteotomy during open-wedge HTO, the tension in the soft tissue envelope is altered by both the osteotomy and the unavoidable complete or partial release of the superficial medial collateral ligament [22]. After surgery, the soft tissue may therefore be vulnerable to medial laxity of the knee joint when bearing the patient's weight, resulting in a slight valgus alignment in standing whole leg radiographs compared with intraoperative corrected alignment [23].

Although several studies have addressed the effect of the navigation system on the accuracy of alignment correction in HTO, most of these studies compared mean postoperative MA and/or WBL ratio of the lower limb. The accuracy and reliability of a monitoring system, such as surgical computer navigation in the operating room, may be more accurately determined by the number of outliers from the acceptable tolerance of the target point than by comparison of mean values [16]. By pooling the results of several studies, this metaanalysis showed that the percent of alignment correction outliers was significantly lower with computer navigated than with conventional HTO. This finding may be explained by the vulnerability of radiographic alignment to knee position. Errors in alignment correction may be due to both inaccurate preoperative planning and inadequate intraoperative quantitative assessment of the amount of correction, both of which are assessed radiographically or by intraoperative fluoroscopy, which are dependent on correct positioning. Measurement errors due to knee flexion and axial rotation can also occur at the moment a radiograph is taken. Coronal alignment of the knee could be affected by these flexion contractures and/or malrotations $[24,25]$.
Posterior tibial slope has been reported to increase inadvertently after open-wedge HTO due to the distinguishing anatomical features of the cross-sectional shape of the proximal tibia [26-28]. Four of the studies included in this metaanalysis compared the change in slope from before to after surgery in patients who underwent computer navigated and conventional HTO, finding that posterior slope after surgery was slightly greater using both methods. The meta-analysis, however, found that changes in posterior slope were similar in the two groups. Posterior tibial slope was not monitored in all studies of computer navigation systems included in this meta-analysis. Change in tibial slope following openwedge HTO may be influenced by several factors, including the completeness of posterior corticotomy, the release of posterior soft tissue, the location of the plate and the lateral hinge, and the ratio of anterior to posterior gaps at the osteotomy site [29, 30]. Advanced versions of computer navigation systems are needed which include protocols that address the inability of current systems to control posterior slope.

This study had several limitations. All of the studies included in this meta-analysis were observational comparison studies. Therefore, there was some inherent heterogeneity due to uncontrolled bias. In addition, there were some differences among studies in surgical techniques (e.g., partial release or complete resection of the medial collateral ligament), acceptable range of alignment correction from the target point, and tool used to measure radiographic parameters (e.g., manual ruler on conventional radiographs or calibrated program on digital radiographs). These factors may explain, at least in part, some of the heterogeneities in the results of this meta-analysis. Another limitation was that the same computer navigation system was used in 9 of the 10 included studies, which may have led to selection bias.

In conclusion, compared with conventional open-wedge HTO, computer navigated open-wedge HTO resulted in slightly more valgus coronal alignment and more reliable lower limb alignment correction within the target range but had no effect on change of posterior tibial slope.

\section{Disclosure}

Level of evidence is meta-analysis (Level II).

\section{Conflicts of Interest}

The authors declare that there are no conflicts of interest regarding the publication of this paper.

\section{Authors' Contributions}

Seung-Beom Han and Hyun Jung Kim contributed equally to this work.

\section{Acknowledgments}

This study was supported by SMC-Ottogi Research Fund (SMX1162171). 


\section{References}

[1] S. Hankemeier, T. Hufner, G. Wang et al., "Navigated openwedge high tibial osteotomy: Advantages and disadvantages compared to the conventional technique in a cadaver study," Knee Surgery, Sports Traumatology, Arthroscopy, vol. 14, no. 10, pp. 917-921, 2006.

[2] F. R. Noyes, W. Mayfield, S. D. Barber-Westin, J. C. Albright, and T. P. Heckmann, "Opening wedge high tibial osteotomy: An operative technique and rehabilitation program to decrease complications and promote early union and function," American Journal of Sports Medicine, vol. 34, no. 8, pp. 1262-1273, 2006.

[3] P. J. Demeo, E. M. Johnson, P. P. Chiang, A. M. Flamm, and M. C. Miller, "Midterm follow-up of opening-wedge high tibial osteotomy," American Journal of Sports Medicine, vol. 38, no. 10, pp. 2077-2084, 2010.

[4] D. C. Lee and S. J. Byun, "High Tibial Osteotomy," Knee Surgery \& Related Research, vol. 24, no. 2, pp. 61-69, 2012.

[5] T.-W. Huang, K.-T. Peng, K.-C. Huang, M. S. Lee, and R. W.-W. Hsu, "Differences in component and limb alignment between computer-assisted and conventional surgery total knee arthroplasty," Knee Surgery, Sports Traumatology, Arthroscopy, vol. 22, no. 12, pp. 2954-2961, 2014.

[6] S. C. Leek, K. A. Jung, C. H. Nam, S. H. Jung, and S. H. Hwang, "The short-term follow-up results of open wedge high tibial osteotomy with using an Aescula open wedge plate and an allogenic bone graft: The minimum 1-year follow-up results," Clinics in Orthopedic Surgery, vol. 2, no. 1, pp. 47-54, 2010.

[7] S. Han, J. Bae, S. Lee et al., "Biomechanical Properties of a New Anatomical Locking Metal Block Plate for Opening Wedge High Tibial Osteotomy: Uniplane Osteotomy," Knee Surgery \& Related Research, vol. 26, no. 3, pp. 155-161, 2014.

[8] D.-H. Lee, J.-H. Park, D.-I. Song, D. Padhy, W.-K. Jeong, and S.-B. Han, "Accuracy of soft tissue balancing in TKA: Comparison between navigation-assisted gap balancing and conventional measured resection," Knee Surgery, Sports Traumatology, Arthroscopy, vol. 18, no. 3, pp. 381-387, 2010.

[9] D.-H. Lee, K.-W. Nha, S.-J. Park, and S.-B. Han, "Preoperative and postoperative comparisons of navigation and radiologic limb alignment measurements after high tibial osteotomy," Arthroscopy - Journal of Arthroscopic and Related Surgery, vol. 28, no. 12, pp. 1842-1850, 2012.

[10] Y. Akamatsu, N. Mitsugi, Y. Mochida et al., "Navigated opening wedge high tibial osteotomy improves intraoperative correction angle compared with conventional method," Knee Surgery, Sports Traumatology, Arthroscopy, vol. 20, no. 3, pp. 586-593, 2012.

[11] R. Iorio, A. Vadalà, S. Giannetti et al., "Computer-assisted high tibial osteotomy: preliminary results.," Orthopedics, vol. 33, no. 10, pp. 82-86, 2010.

[12] S.-J. Kim, Y.-G. Koh, Y.-M. Chun, Y.-C. Kim, Y.-S. Park, and C.H. Sung, "Medial opening wedge high-tibial osteotomy using a kinematic navigation system versus a conventional method: A 1-year retrospective, comparative study," Knee Surgery, Sports Traumatology, Arthroscopy, vol. 17, no. 2, pp. 128-134, 2009.

[13] D.-H. Lee, S.-B. Han, K.-J. Oh et al., "The weight-bearing scanogram technique provides better coronal limb alignment than the navigation technique in open high tibial osteotomy," Knee, vol. 21, no. 2, pp. 451-455, 2014.

[14] J. Lutzner, A. F. Gross, K. P. Gunther, and S. Kirschner, "Precision of navigated and conventional open-wedge high tibial osteotomy in a cadaver study," European Journal of Medical Research, vol. 15, no. 3, pp. 117-120, 2010.

[15] F. Maurer and G. Wassmer, "High tibial osteotomy: Does navigation improve results?” Orthopedics, vol. 29, no. 10, pp. S130-S132, 2006.

[16] K. Reising, P. C. Strohm, O. Hauschild et al., "Computerassisted navigation for the intraoperative assessment of lower limb alignment in high tibial osteotomy can avoid outliers compared with the conventional technique," Knee Surgery, Sports Traumatology, Arthroscopy, vol. 21, no. 1, pp. 181-188, 2013.

[17] C. H. Ribeiro, N. R. Severino, and P. M. Moraes De Barros Fucs, "Opening wedge high tibial osteotomy: Navigation system compared to the conventional technique in a controlled clinical study," International Orthopaedics, vol. 38, no. 8, pp. 1627-1631, 2014.

[18] D. Saragaglia and J. Roberts, "Navigated osteotomies around the knee in 170 patients with osteoarthritis secondary to genu varum," Orthopedics, vol. 28, no. 10, pp. s1269-s1274, 2005.

[19] C. H. Ribeiro, N. R. Severino, and P. M. De Moraes Barros Fucs, "Preoperative surgical planning versus navigation system in valgus tibial osteotomy: A cross-sectional study," International Orthopaedics, vol. 37, no. 8, pp. 1483-1486, 2013.

[20] T. W. Dugdale, F. R. Noyes, and D. Styer, "Preoperative planning for high tibial osteotomy: The effect of lateral tibiofemoral separation and tibiofemoral length," Clinical Orthopaedics and Related Research, no. 274, pp. 248-264, 1992.

[21] D. K. Bae, J. W. Lee, S. J. Cho, and S. J. Song, "Comparison of Mechanical Axis and Dynamic Range Assessed with Weight Bearing Radiographs and Navigation System in Closed Wedge High Tibial Osteotomy," Knee Surgery \& Related Research, vol. 29, no. 3, pp. 231-237, 2017.

[22] J. A. Sim, J. H. Kwak, S. H. Yang, E. S. Choi, and B. K. Lee, "Effect of weight-bearing on the alignment after open wedge high tibial osteotomy," Knee Surgery, Sports Traumatology, Arthroscopy, vol. 18, no. 7, pp. 874-878, 2010.

[23] D. Kendoff, T. N. Board, M. Citak et al., "Navigated lower limb axis measurements: Influence of mechanical weight-bearing simulation," Journal of Orthopaedic Research, vol. 26, no. 4, pp. 553-561, 2008.

[24] D. Kendoff, M. Citak, A. Pearle et al., "Influence of lower limb rotation in navigated alignment analysis: Implications for high tibial osteotomies," Knee Surgery, Sports Traumatology, Arthroscopy, vol. 15, no. 8, pp. 1003-1008, 2007.

[25] H. Kawakami, N. Sugano, K. Yonenobu et al., "Effects of rotation on measurement of lower limb alignment for knee osteotomy," Journal of Orthopaedic Research, vol. 22, no. 6, pp. 1248-1253, 2004.

[26] F. R. Noyes, S. X. Goebel, and J. West, "Opening wedge tibial osteotomy: The 3-triangle method to correct axial alignment and tibial slope," American Journal of Sports Medicine, vol. 33, no. 3, pp. 378-387, 2005.

[27] S. W. O. Moon, S. H. Y. Park, B. H. O. Lee et al., "The Effect of Hinge Position on Posterior Tibial Slope in Medial Open-Wedge High Tibial Osteotomy," Arthroscopy : the journal of arthroscopic \& related surgery : official publication of the Arthroscopy Association of North America and the International Arthroscopy Association, vol. 31, no. 6, pp. 1128-1133, 2015.

[28] S. Seo, O. Kim, J. Seo, D. Kim, Y. Kim, and I. Lee, "Complications and Short-Term Outcomes of Medial Opening Wedge High Tibial Osteotomy Using a Locking Plate for Medial Osteoarthritis of the Knee," Knee Surgery \& Related Research, vol. 28, no. 4, pp. 289-296, 2016. 
[29] E.-K. Song, J.-K. Seon, and S.-J. Park, "How to avoid unintended increase of posterior slope in navigation-assisted open-wedge high tibial osteotomy," Orthopedics, vol. 30, no. 10, pp. S127-S131, 2007.

[30] E.-K. Song, J.-K. Seon, S.-J. Park, and H.-Y. Seo, "Navigated open wedge high tibial osteotomy," Sports Medicine and Arthroscopy Review, vol. 16, no. 2, pp. 84-90, 2008. 


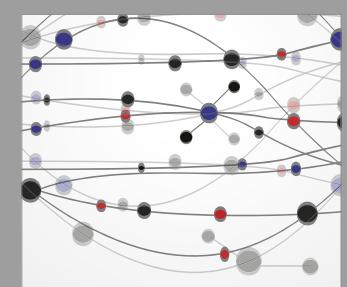

The Scientific World Journal
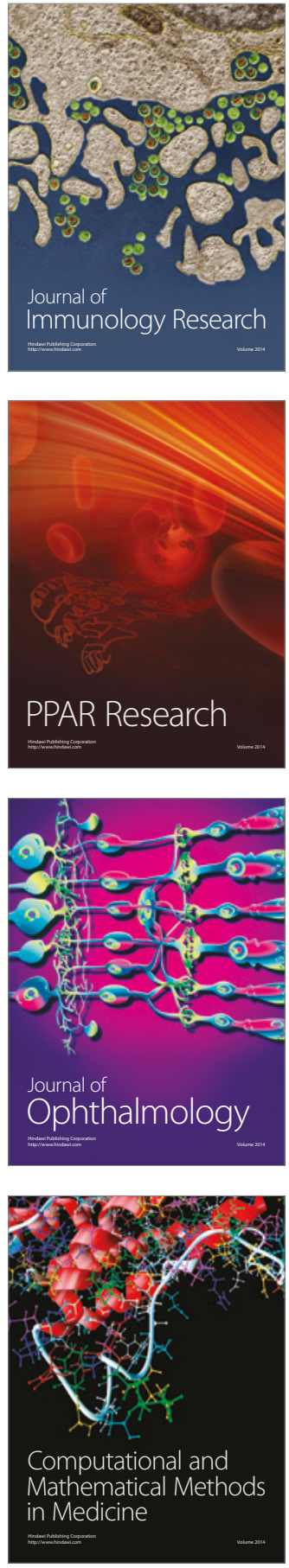

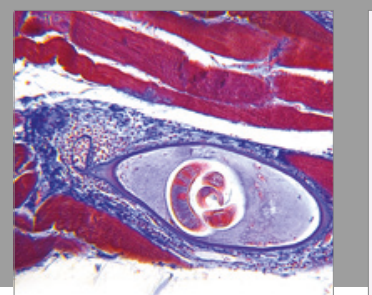

Gastroenterology Research and Practice
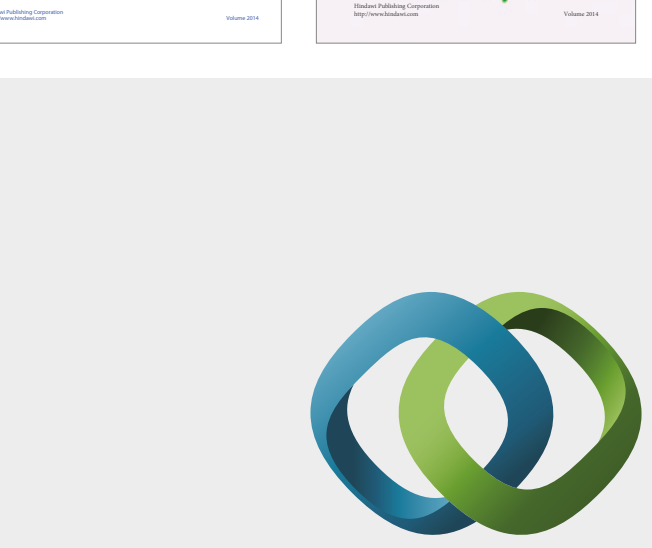

\section{Hindawi}

Submit your manuscripts at

https://www.hindawi.com
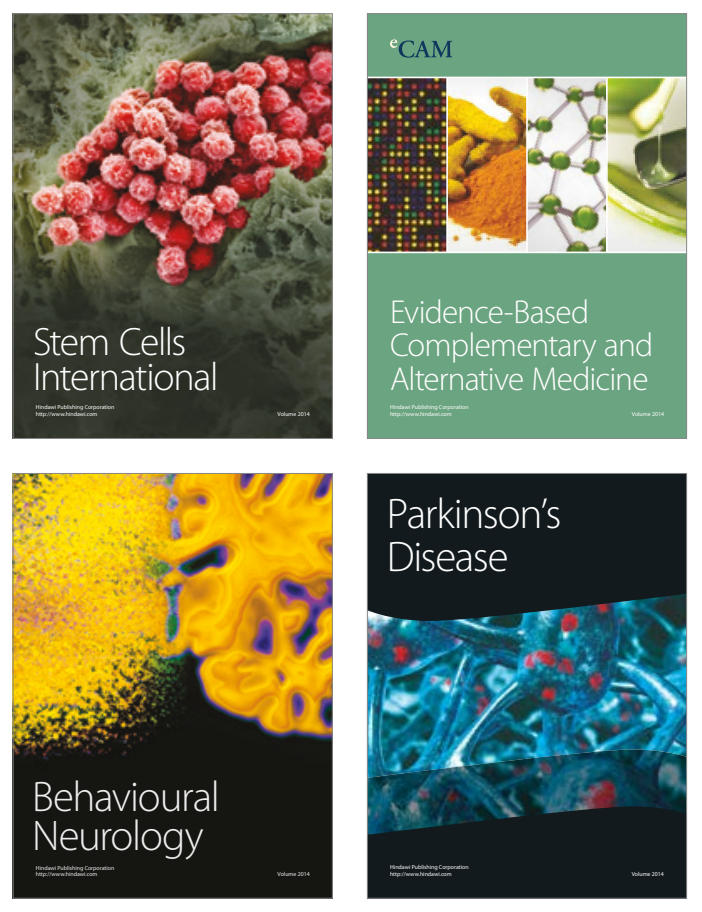
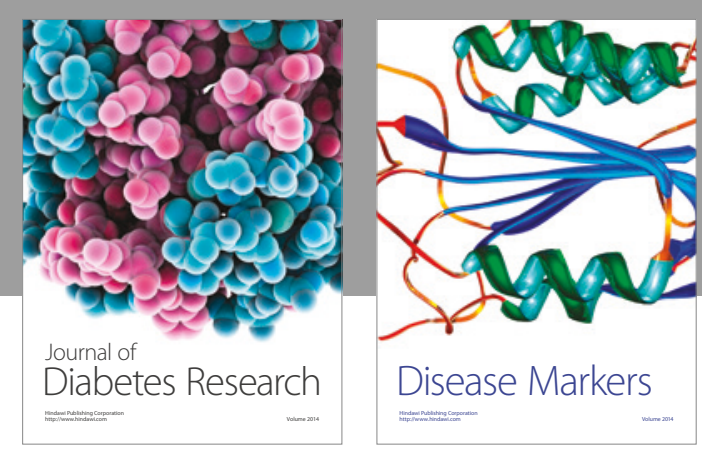

Disease Markers
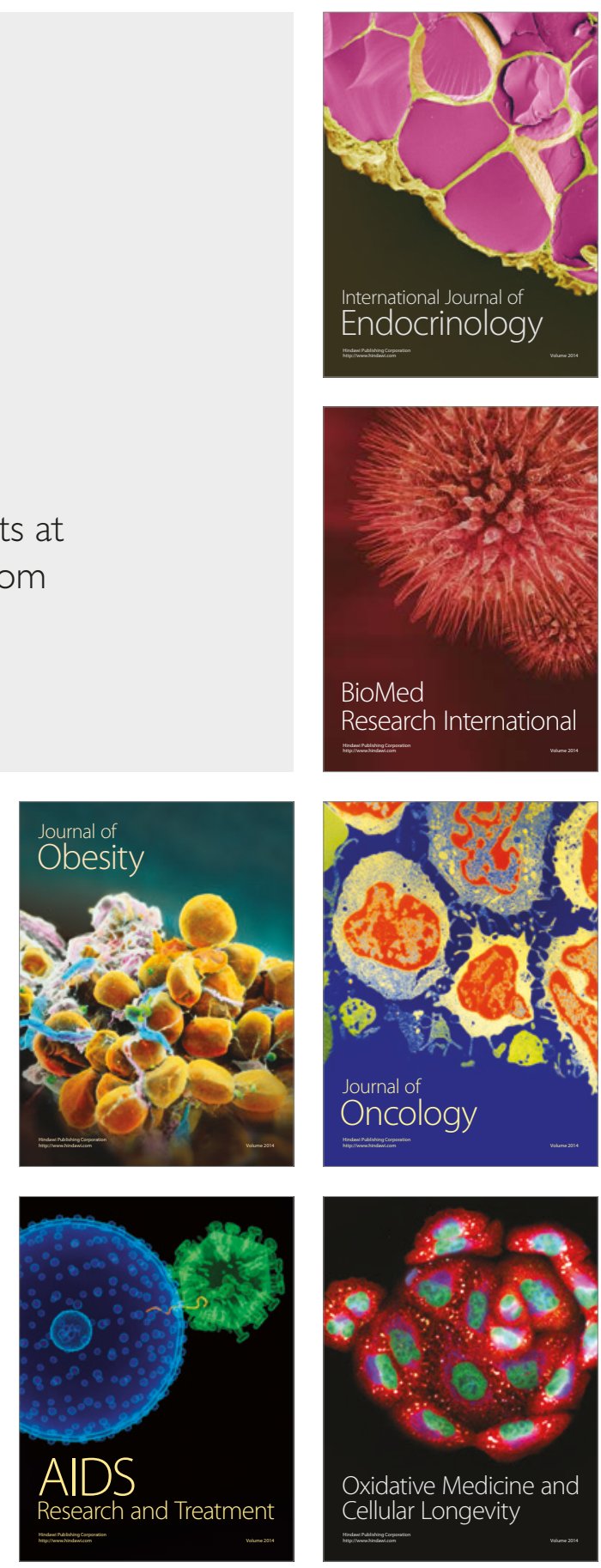